\title{
DTL promotes melanoma progression through rewiring cell glucose metabolism
}

\author{
Jia-Jing Lu\#, Fu-Juan Chen", Ying Li", Xin Xu, Chen Peng, Ning Yu, Li-Na Su, Li Tang \\ Department of Dermatology, Shanghai Skin Disease Hospital, Tongji University School of Medicine, Shanghai, China \\ Contributions: (I) Conception and design: JJ Lu, FJ Chen, Y Li, LN Su, L Tang; (II) Administrative support: LN Su, L Tang; (III) Provision of study \\ materials or patients: JJ Lu, FJ Chen, Y Li; (IV) Collection and assembly of data: Y Li, X Xu, C Peng, N Yu; (V) Data analysis and interpretation: JJ \\ Lu, FJ Chen, Y Li, L Tang; (VI) Manuscript writing: All authors; (VII) Final approval of manuscript: All authors. \\ \#These authors contributed equally to this work. \\ Correspondence to: Li Tang; Li-Na Su. Department of Dermatology, Shanghai Skin Disease Hospital, Tongji University School of Medicine, 1278 \\ Baode Road, Shanghai 200443, China. Email: jhcn@163.com; sulina11@hotmail.com.
}

\begin{abstract}
Background: To investigate the role of DTL in the development of skin cutaneous melanoma (SKCM) and possible mechanisms.

Methods: We examined the expression of DTL in SKCM in The Cancer Genome Atlas (TCGA) and Oncomine database and analyzed the relationship between DTL expression and melanoma prognosis. Furthermore, we silenced the DTL gene by RNA interference in A375 cells and investigated the effect of DTL silencing on the biological function of melanoma cells.

Results: The expression of DTL in SKCM was upregulated in the tumor tissues compared with the paired normal tissues. Survival analysis showed that higher DTL expression in SKCM patients was associated with poor clinical outcome compared with the lower DTL expression group. Silencing of DTL in A375 cells significantly inhibited the melanoma cell growth and proliferation ability, and also significantly decreased the total glucose consumption and lactate production. Gene set enrichment analysis (GSEA) showed that MYC targets gene set pathway was highly enriched in the DTL high expression group. The expression levels of some MYC targets-related oncogenes, including c-MYC, HK1, HK2, PGK1, ENO1, LDHA, IDH1, ACLY, and $H M G C R$, were reduced in the A375 cells with knockdown DTL and upregulated in SKCM tissues with high DTL expression, and there was a positive correlation between them.
\end{abstract}

Conclusions: An important role is played by DTL in promoting melanoma cell growth and glucose metabolism, possibly through activation of the $M Y C$ target pathway.

Keywords: Skin cutaneous melanoma (SKCM); DTL; glucose metabolism

Submitted Nov 23, 2021. Accepted for publication Jan 07, 2022.

doi: $10.21037 /$ atm-21-6648

View this article at: https://dx.doi.org/10.21037/atm-21-6648

\section{Introduction}

Skin cutaneous melanoma (SKCM) is the most malignant skin cancer, accounting for more than $75 \%$ of skin cancer deaths (1). Despite great advances in targeted therapy and immunotherapy for SKCM over the past decade, the overall survival (OS) rate for SKCM remains low, especially for advanced SKCM. Therefore, exploring the mechanisms of SKCM development is of great benefit to understand the biological behavior of SKCM and to find new treatments.

The DTL gene, also known as CDT2, DCAF2, or $R A M P$, is a denticleless $\mathrm{E} 3$ ubiquitin protein ligase. It plays an important role in regulating CDT1 proteolysis, DNA replication, and cell cycle (2,3). Functional abnormalities of DTL are involved in the development of many human malignancies. Elevated DTL expression has been found in a variety of human malignancies including breast cancer, colorectal cancer, lung cancer, and hepatocellular 
carcinoma, and elevated $D T L$ expression is positively associated with poor prognosis of these malignancies (4-7). In in vitro experiments, silencing DTL could significantly impair tumor cell growth and invasion capability, and induce apoptosis (8). The DTL gene is also critical in the development of SKCM. It has been found to be frequently overexpressed in SKCM tissues, and its overexpression has been associated with poor outcome $(9,10)$. In vitro analysis has demonstrated that silencing DTL could inhibit melanoma cell proliferation via inducing DNA rereplication and senescence (10). However, the role and mechanisms of $D T L$ in SKCM are still far from being elucidated.

In this study, we examined the expression of DTL in SKCM in The Cancer Genome Atlas (TCGA) and Oncomine database and analyzed the relationship between DTL expression and SKCM prognosis. Furthermore, we silenced the $D T L$ gene by RNA interference in a melanoma cell line and investigated the effect of DTL silencing on the biological function of melanoma cells. Our aim was to reveal the role and possible mechanisms of DTL in melanoma and to initially establish an association between DTL and melanoma cell metabolic rewiring which shows good promise in the treatment of melanoma.

We present the following article in accordance with the MDAR reporting checklist (available at https://atm. amegroups.com/article/view/10.21037/atm-21-6648/rc).

\section{Methods}

\section{DTL expression analysis from the TCGA and Oncomine database}

The messenger RNA (mRNA) expression levels of DTL in SKCM patient tissues were analyzed from TCGA (https:// tcga-data.nci.nih.gov/publications/tcga) and Oncomine database (https://www.oncomine.org/resource/main.html). A total of 461 SKCM tumor specimens and 558 normal tissues were available from TCGA normal, GenotypeTissue Expression (GTEx) data, and the Oncomine cohorts including the Riker melanoma ( $\mathrm{n}=14$, tumor tissues; $\mathrm{n}=4$, normal tissues) and Talantov melanoma $(n=45$, tumor tissues; $\mathrm{n}=7$, normal tissues). The study was conducted in accordance with the Declaration of Helsinki (as revised in 2013).

\section{Kaplan-Meier survival analysis of the prognostic value of DTL}

We obtained SKCM patient tissues containing patient survival information from TCGA database (https://tcgadata.nci.nih.gov/publications/tcga). In line with the DTL expression median value in the SKCM patient tissues, they were classified into two groups: DTL high expression group and $D T L$ low expression group.

\section{Cell culture and stable cell lines}

The A375 cell line was obtained from the Cell Bank of Shanghai Institutes of Biological Sciences, Chinese Academy of Sciences (Shanghai, China). The A375 cells were passaged in Dulbecco's modified Eagle's medium (DMEM; Life Technologies, Carlsbad, CA, USA) supplemented with $10 \%$ fetal bovine serum (FBS; Life Technologies), $1 \times$ penicillin/streptomycin, and $4 \mathrm{mM}$ $\mathrm{L}$-glutamine and incubated at $37{ }^{\circ} \mathrm{C}$ in $5 \% \mathrm{CO}_{2}$. To knockdown the expression of DTL, short hairpin RNA (shRNA; shDTL-1: GCCTAGTAACAGTAACGAGTA and shDTL-2: CTGGTGAACTTAAACTTGTTA) was cloned in pLKO.1 plasmid. For lentivirus production, HEK293T cells were transfected with the lentiviral vectors and helper plasmids DR8.91 and VSVG. At $48 \mathrm{~h}$ after transfection, virus-containing media were collected. The A375 cells were transduced using lentivirus for $24 \mathrm{~h}$, and then selected with puromycin.

\section{Cell proliferation assay}

Cell proliferation was measured with a Cell Counting Kit-8 (CCK-8) kit. The A375 cells stably expressing vector (A375SCR) or shDTL (A375-shDTL-1 and A375-shDTL-2) (3,000 cells/well) were seeded into 96-well plates. The A375-SCR, A375-shDTL-1, and A375-shDTL-2 cells were cultured at $37{ }^{\circ} \mathrm{C}$ in $5 \% \mathrm{CO}_{2}$ for $24,48,72$, and $96 \mathrm{~h}$, then the medium of each well was replaced with $10 \%$ CCK8 solution in fresh medium. The above cells were then incubated for $2 \mathrm{~h}$, following which absorbance at $450 \mathrm{~nm}$ was measured using a microplate reader.

\section{Colony formation assay}

The A375-SCR, A375-shDTL-1, and A375-shDTL-2 cells were seeded respectively in 6-well plates at a density of 200 cells/well at $37{ }^{\circ} \mathrm{C}$ in a $5 \% \mathrm{CO}_{2}$ humidified environment. After incubation for about 14 days, the plates were washed with phosphate-buffered saline (PBS) and fixed with methanol for $30 \mathrm{~min}$, then stained with Giemsa solution (Sigma-Aldrich, St. Louis, MO, USA) for $30 \mathrm{~min}$. 
Afterwards, the plates were washed with clean water and the number of the above cell colonies were counted.

\section{Gene set enrichment analysis (GSEA) of DTL}

GSEA was performed using RNA-seq data sets from TCGA-SKCM (https://tcga-data.nci.nih.gov/publications/ TCGA-SKCM). According to the mRNA expression median level of DTL gene in the SKCM patient tissues, the tissues were divided into two groups: DTL high expression group (DTL-high, $\mathrm{n}=236$ ) and DTL low expression group (DTL-low, n=237).

\section{Statistical analysis}

Statistical analysis was performed with the software SPSS 20 (IBM Corp., Armonk, NY, USA). Kaplan-Meier survival analysis, Pearson's test, and Student's $t$-test were used to compare the difference of the variables. A $\mathrm{P}$ value $<0.05$ was considered statistically significant.

\section{Results}

\section{Upregulation of DTL in SKCM is associated with worse clinical outcomes}

To reveal the clinical relevance of DTL in human SKCM, we analyzed the expression of DTL in the SKCMTCGA database and found that DTL mRNA levels were significantly increased in the SKCM tumor tissues compared with the normal adjacent tissues (Figure 1A). Moreover, we further examined the expression of DTL in the SKCM data from the Oncomine database and also found that DTL expression in the Riker melanoma and Talantov melanoma were notably upregulated in the tumor tissues compared with the paired normal tissues (Figure 1B,1C), suggesting the potential oncogenic activity of $D T L$. We then analyzed the DTL expression level in tumor tissues derived from 458 post-operative SKCM patients from TCGA database. These SKCM patient tissues were grouped under high DTL expression or low DTL expression according to their $D T L$ mRNA levels. Next, we evaluated the relationship between DTL expression level and patient OS and diseasefree survival (DFS) by Kaplan-Meier survival analysis. Survival analysis revealed that higher DTL expression in SKCM patients was associated with poor clinical outcome as well as shorter DFS times compared with the lower DTL expression group (Figure 1D,1E). Taken together, these results indicated that upregulation of DTL contributes to cancer development and can serve as a novel indicator for poor prognosis in SKCM.

\section{DTL promotes cell growth and glucose metabolism in SKCM}

To evaluate the function of $D T L$ in the progression of SKCM, we firstly constructed the DTL high or low expression SKCM cell lines, knocked down DTL expression in the A375 cells with the lentivirus expressing DTL shRNA precursor (A375-shDTL-1 and -2), and used the realtime polymerase chain reaction (PCR) assay to confirm the knockdown efficiency of DTL in the A375-shDTL-1 and -2 cells compared with the control cells (A375-SCR) (Figure $2 A$ ). The above cells were used to evaluate $D T L$ influence on SKCM progression. Using the CCK-8 assay, we found that knockdown of the expression of DTL in the A375 cells significantly inhibited the cell proliferation ability (Figure 2B). Furthermore, we also revealed that $D T L$ knockdown notably impaired the tumor cell colony formation ability (Figure 2C,2D). We then analyzed glucose metabolism upon DTL knockdown in A375 cells. As expected, DTL silencing significantly decreased total glucose consumption and lactate production in the A375 cells (Figure 2E,2F). Collectively, these results demonstrate that DTL promotes SKCM cell growth and enhances glucose metabolism.

\section{DTL expression levels positively correlate with the MYC targets patbway and SKCM progression}

To explore the mechanism underlying DTL's promotion of SKCM cell growth and glucose metabolism, we firstly made an analysis of the RNA-seq data of SKCM patient tissues $(\mathrm{n}=473)$ from TCGA database, and then divided the into a DTL high expression group (DTL-high, $\mathrm{n}=236$ ) and DTL low expression group (DTL-low, $\mathrm{n}=237$ ). Moreover, we performed GSEA for the RNA-seq data between the DTL-high and DTL-low group, and found that cell cycle and Jaeger metastasis up gene pathways were significantly enriched in the SKCM patient tissues of the DTL high expression group (Figure $3 A, 3 B$ ), which were similar to the results of DTL's promotion of SKCM progression. Furthermore, $M Y C$ targets $\mathrm{V} 1$ and $M Y C$ targets $\mathrm{V} 2$ gene set pathways were also highly enriched in the DTL high expression group (Figure 3C,3D), suggesting DTL may promote glucose metabolism via activating $M Y C$ targets 
A DTL expression in TCGA SKCM

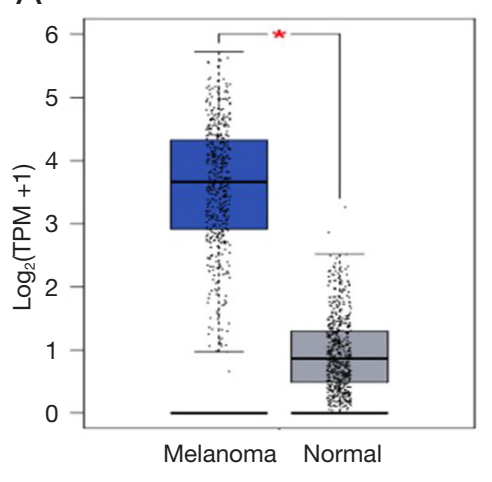

D 1 .

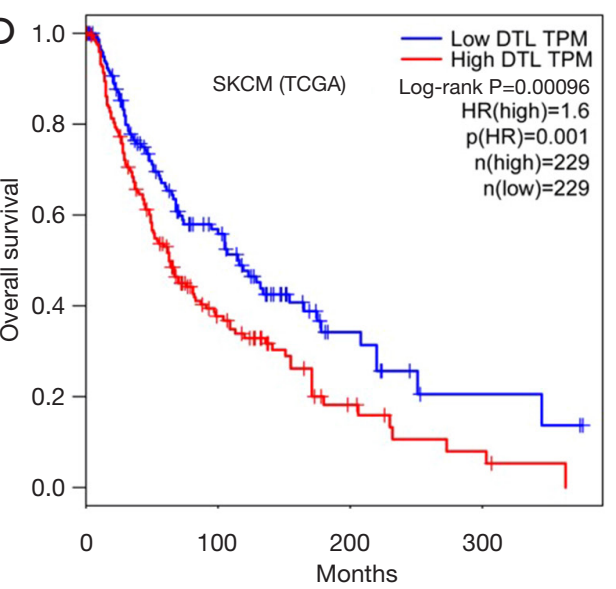

B DTL expression in Riker melanoma

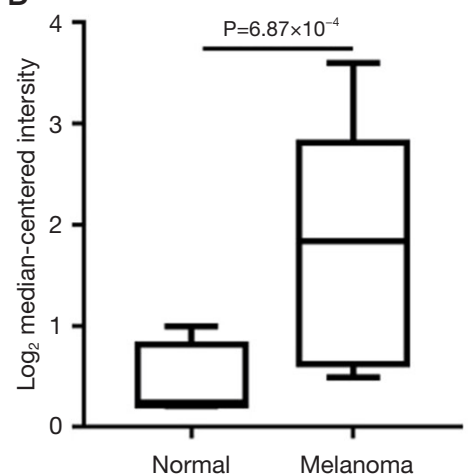

C DTL expression in Talantov melanoma

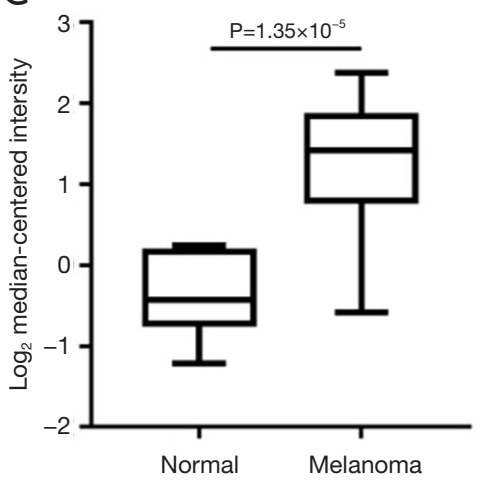

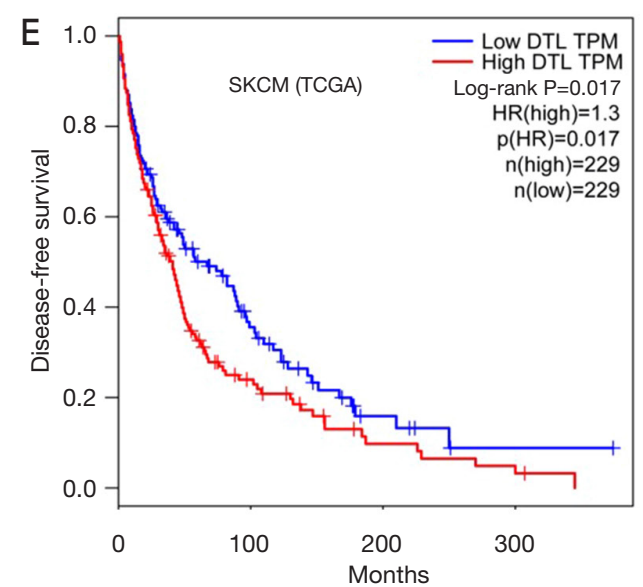

Figure 1 Upregulation of DTL in SKCM is associated with worse clinical outcomes. (A) The expression of DTL between SKCM tumor specimens $(\mathrm{n}=461)$ and 558 normal tissues $(\mathrm{n}=558)$ from the TCGA normal and GTEx data. ${ }^{*} \mathrm{P}<0.01$. (B,C) The mRNA levels of DTL in the Riker melanoma ( $n=14$, tumor tissues; $n=4$, normal tissues) (B) and Talantov melanoma $(n=45$, tumor tissues; $n=7$, normal tissues) (C) from the Oncomine database (https://www.oncomine.org/resource/main.html). The expression of DTL was up-regulated in the melanoma tissues comparing with the normal tissues. (D,E) Kaplan-Meier curves of OS (D) and DFS (E) of SKCM patient according to DTL expression levels in the SKCM tissues. The OS and DFS was significantly reduced in the DTL high expression group (n=229) compared with the DTL low expression group ( $\mathrm{n}=229)$. The SKCM patients data come from TCGA database (https://tcga-data.nci.nih.gov/publications/tcga). Log-rank P values were shown. SKCM, skin cutaneous melanoma; TCGA, The Cancer Genome Atlas; GTEx, Genotype-Tissue Expression; mRNA, messenger RNA; OS, overall survival; DFS, disease-free survival; TPM, transcripts per million; HR, hazard ratio.

V1-2 gene set pathway. In addition, the G2M checkpoint, $\mathrm{E} 2 \mathrm{~F}$ targets, mitotic spindle, and mTORC1 signaling gene set pathways were notably enriched in the DTL high expression group compared with the DTL low expression group (Figure $3 E-3 H$ ). Thus, these findings indicate that $D T L$ promotes the MYC targets pathway and SKCM progression.

\section{DTL drives the cell glucose metabolism reprogramming in SKCM}

Next, we investigated whether DTL could active the
$M Y C$ targets pathway to promote tumor cell glucose metabolism reprogramming. Firstly, we detected several $M Y C$ targets-related oncogenes expressions ( $c-M Y C, H K 1$, HK2, PGK1, ENO1, LDHA, IDH1, ACLY, and HMGCR) in the DTL knockdown A375 cells and the real-time PCR results showed that knockdown of the expression of DTL could reduce the above genes' mRNA levels (Figure 4A). Moreover, we also found that $c-M Y C, H K 1, H K 2, P G K 1$, ENO1, LDHA, IDH1, ACLY, and HMGCR expression levels were upregulated in the DTL high expression SKCM patient tissues compared with the DTL low expression group (Figure $4 B$ ). Furthermore, a positive correlation 
A

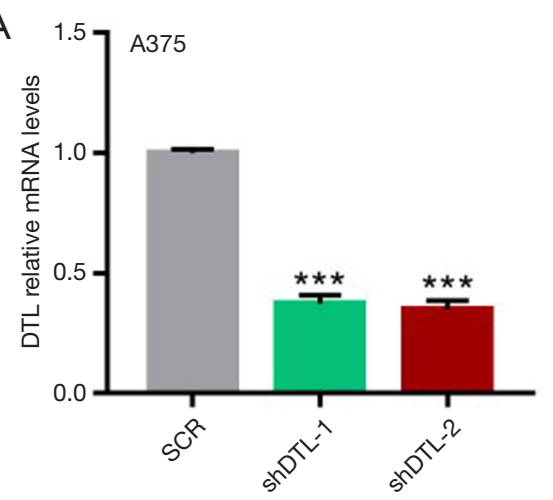

C

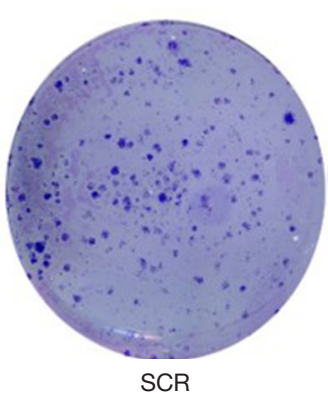

SCR

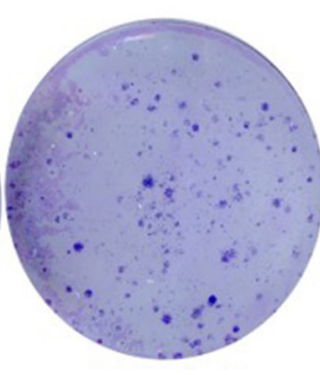

shDTL-1

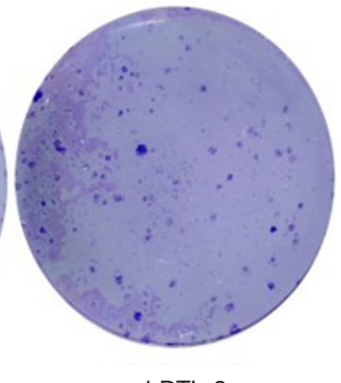

shDTL-2

B

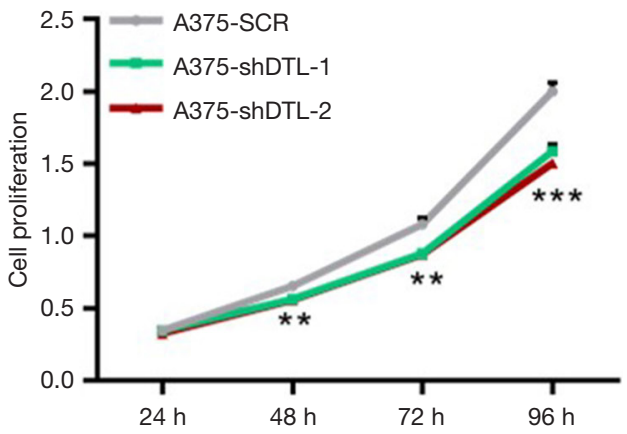

D
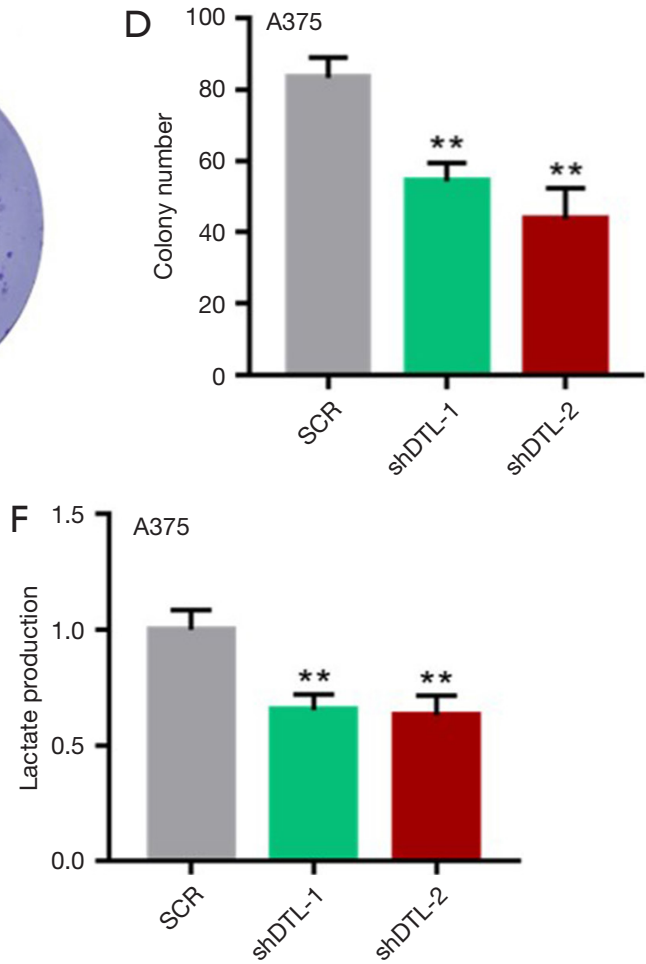

Figure 2 DTL promotes cell growth and glucose metabolism in SKCM. (A) Knockdown expression of DTL in the A375 cells infected with lentivirus as indicated. The efficiency of DTL knockdown in the A375 cells were assessed by real-time PCR. (B) Using the CCK-8 assays to examine the A375 cell proliferation ability as the indicated. (C,D) The cell colony formation assay of A375 cells infected with shRNA targeting DTL and the control (Giemsa staining). (E,F) Glucose consumption (E) and lactate production (F) of A375 cells infected with shRNA targeting DTL (shDTL-1 and -2) were determined. The data represent the mean \pm SD. Significant differences were determined using two-tailed Student's $t$-test, ${ }^{*} \mathrm{P}<0.05 ;{ }^{* *} \mathrm{P}<0.01 ;{ }^{* * *} \mathrm{P}<0.001$. SKCM, skin cutaneous melanoma; PCR, polymerase chain reaction; CCK8, Cell Counting Kit-8; shRNA, short hairpin RNA; SD, standard deviation.

was found between the DTL expression and $c-M Y C, H K 1$, HK2, PGK1, ENO1, LDHA, IDH1, ACLY, and HMGCR expression levels in the SKCM tissues from TCGA database (Figure $4 C-4 K$ ). These results suggest that DTL promotes cell glucose metabolism reprogramming in both SKCM tissues and cell lines.

\section{Discussion}

This study illustrated that DTL expression levels are significantly higher in SKCM tissues than in normal tissues, and that OS and DFS are significantly lower in SKCM patients with high DTL expression than in those with low 

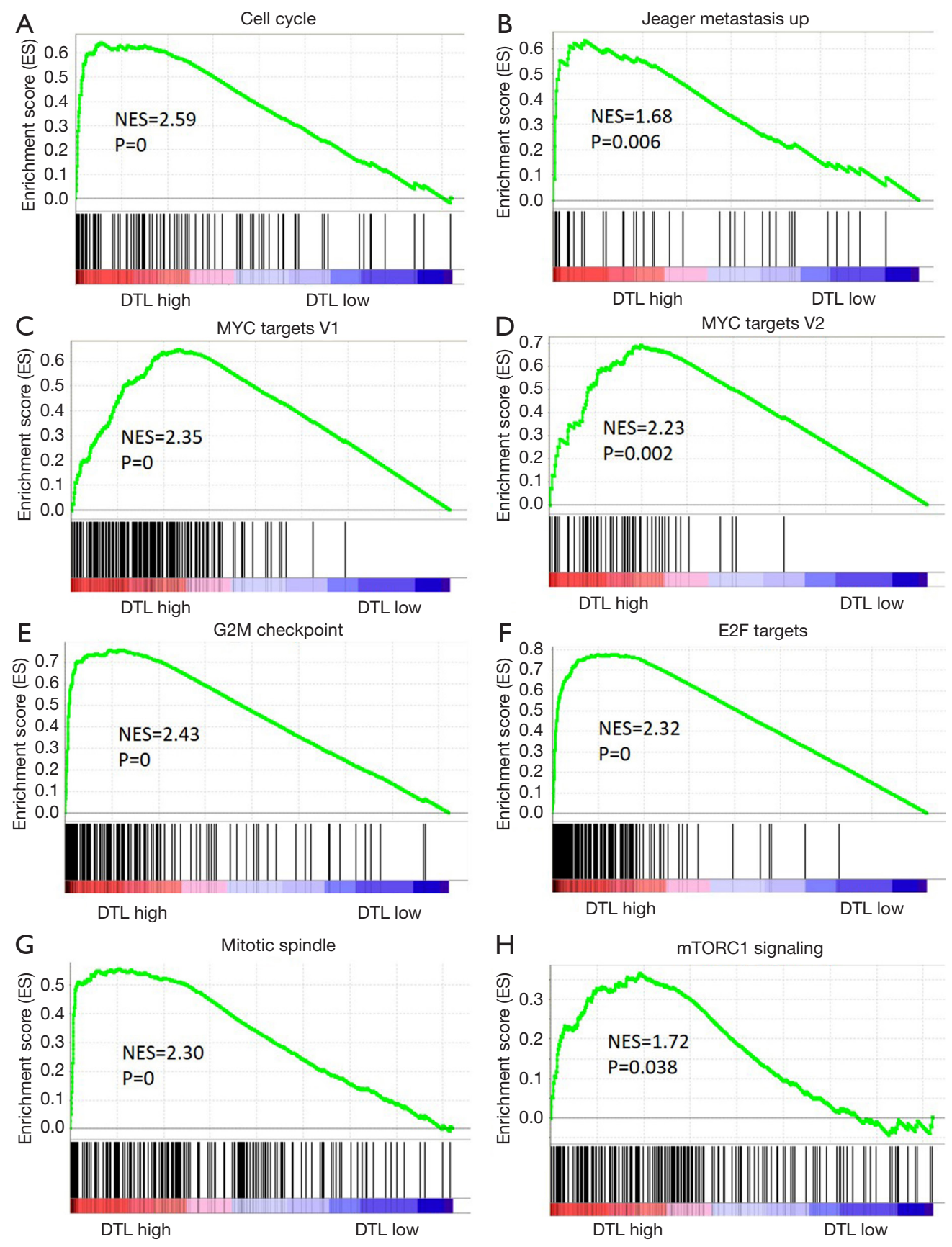

Figure 3 DTL expression levels positively correlates with the MYC targets pathway and SKCM progression. (A-H) Analysis the RNA-seq data of SKCM patient tissues from TCGA, according to the mRNA expression median level of DTL gene in the SKCM patient tissues, which were divided into two groups: DTL high expression group (DTL-high, n=236) and DTL low expression group (DTL-low, n=237). and then followed using GSEA analysis. GSEA analysis indicated that pathways including cell cycle (A), Jaeger metastasis up (B), MYC targets V1 (C), MYC targets V2 (D), G2M checkpoint (E), E2F targets (F), mitotic spindle (G), and mTORC1 signaling (H) were highly enriched in the SKCM patient tissues with DTL high expression group (DTL-high) compared with the DTL low expression group (DTLlow). SKCM, skin cutaneous melanoma; TCGA, The Cancer Genome Atlas; mRNA, messenger RNA; GSEA, gene set enrichment analysis; NES, normalized enrichment score. 

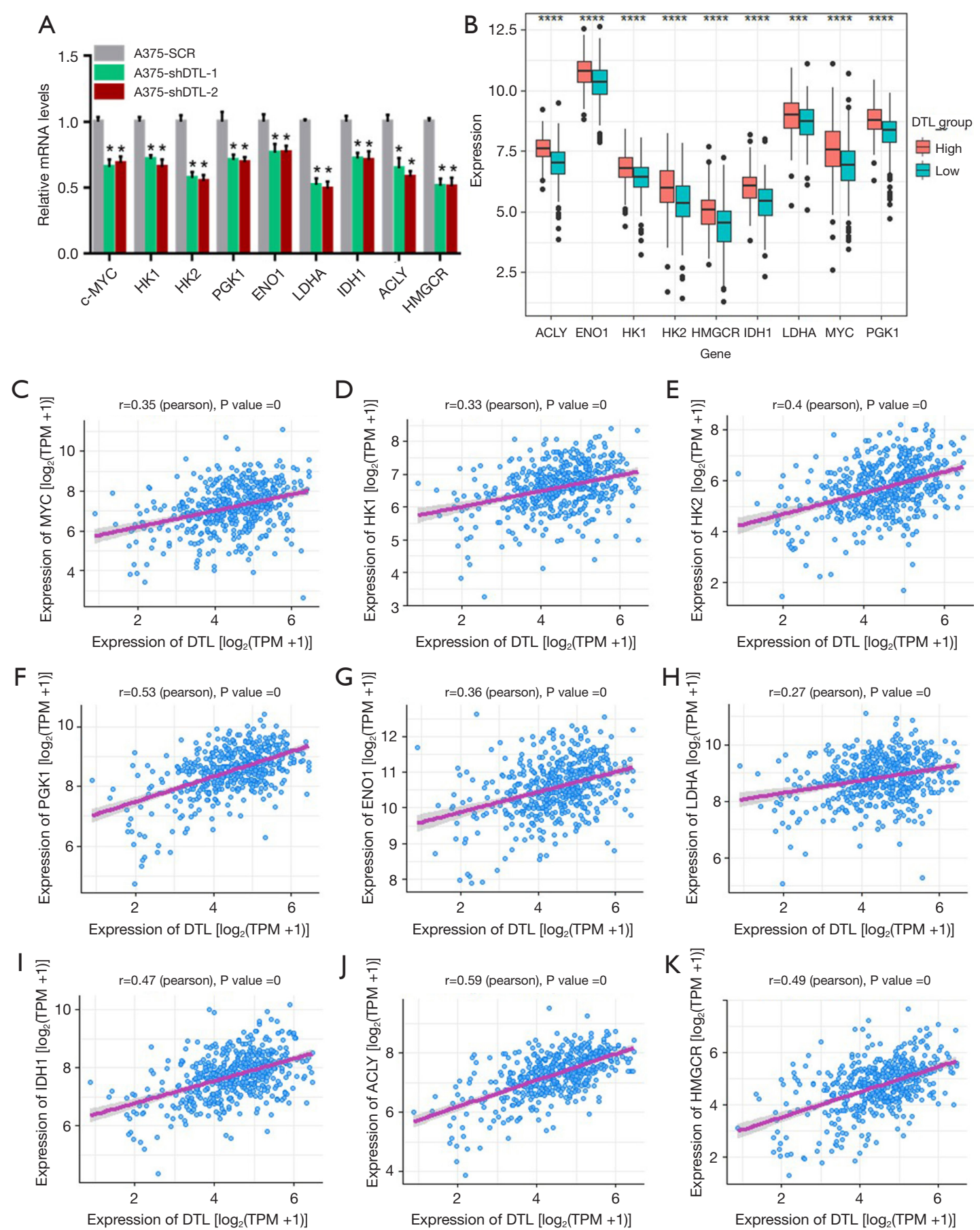

Figure 4 DTL drives the cell glucose metabolism reprogramming in SKCM. (A) The expression of $c-M Y C, H K 1, H K 2, P G K 1, E N O 1$, $L D H A, I D H 1, A C L Y$ and HMGCR in the A375 cells were measured using the real-time PCR. Means \pm SD from three independent experiments were presented. Significant differences were determined using Student's $t$-test, ${ }^{*} \mathrm{P}<0.05$. (B) The expression levels of $c$ - $M Y C$, HK1, HK2, PGK1, ENO1, LDHA, IDH1, ACLY, and HMGCR between in the DTL high expression group (DTL-high, n=236) and DTL low expression group (DTL-low, $\mathrm{n}=237$ ) from SKCM-TCGA database. ${ }^{* * *} \mathrm{P}<0.001$; ${ }^{* * *} \mathrm{P}<0.0001$. (C-K) A positive correlation were found between the levels of $c-M Y C(\mathrm{C}), H K 1$ (D), HK2 (E), PGK1 (F), ENO1 (G), LDHA (H), IDH1 (I), ACLY (J), and HMGCR (K) with DTL expression in the SKCM tissues. The SKCM tissues data come from TCGA. SKCM, skin cutaneous melanoma; PCR, polymerase chain reaction; SD, standard deviation; TCGA, The Cancer Genome Atlas; mRNA, messenger RNA; TPM, transcripts per million. 
DTL expression. In vitro experiments showed that silencing DTL could significantly impair the growth ability and invasive ability of melanoma cells. Silencing DTL also significantly reduced glucose consumption and lactate production in melanoma cells. These findings suggest that DTL can promote the growth and glucose metabolism of melanoma cells and play a vital role in the development of melanoma.

Abnormal glucose metabolism is one of the important features of cancer and plays an important role in promoting the development of melanoma (11). Studies have shown that melanoma cells have abnormally high levels of glucose metabolism, producing large amounts of lactic acid while consuming large amounts of glucose. Melanoma cells, especially the more metastatic ones, are able to take in excess lactic acid, which allows them to increase the production of antioxidants, thus contributing to their survival in the bloodstream $(12,13)$. These metabolic alterations facilitate malignant tumor proliferation and adaptation to unfavorable survival environments, thereby promoting disease progression. Enhanced metabolic activity of tumor cells can also resist melanoma response to adoptive $\mathrm{T}$ cell therapy, which is one of the important reasons for the failure of melanoma immunotherapy (14). Therefore, limiting the metabolic pathways of melanoma cell growth would provide a basis for targeted metabolic therapy for melanoma. In this study, we found that inhibition of DTL expression significantly attenuated the glucose consumption level as well as the lactate production level in melanoma, suggesting that $D T L$ is also involved in the glucose metabolic process of melanoma and that it may promote melanoma progression by altering the its glucose metabolic process. This needs to be supported by more studies.

Metabolic rewiring is an adaptive response process of tumor cells in a low nutrient and hypoxic conditions in the tumor microenvironment (15). Studies have shown that many signaling pathways are partially involved in the process of metabolic changes through genetic and epigenetic alterations. These signaling pathways and molecules include the RAS/MAPK pathway, the AKT pathway, $R S K$ and SOX4 $(16,17)$. Targeting metabolic rewiring process could increase the efficiency of target therapy and the sensitivity to immunotherapy in melanoma (18). Thus, the discovery of additional molecules that regulate metabolic rewiring offers promising prospects for therapies that target metabolic rewiring. The present study mainly provides an association between DTL and metabolic rewiring, although the pathway through which $D T L$ promotes melanoma glucose metabolism is currently unknown. As a ubiquitin ligase, $D T L$ may act by the ubiquitination pathway of degrading tumor suppressor proteins or by directly activating related oncogene pathways. In this study, we found that $M Y C$ targets gene set pathway was highly enriched in the DTL high expression group, and inhibition of DTL expression could downregulate the expression levels of some important $M Y C$ targets-related oncogenes. In addition, the expression levels of $M Y C$ targets-related oncogenes including $c-M Y C$, HK1, HK2, PGK1, ENO1, LDHA, IDH1, ACLY, and HMGC were upregulated in SKCM tissues with high expression of DTL, whereas the expression levels of the above $M Y C$ targets-related oncogenes were downregulated in melanoma cell lines with knockdown of DTL. These results suggest that DTL may promote melanoma cell glucose metabolism reprogramming by activating $M Y C$ target pathway.

In summary, our study revealed that DTL plays an important role in promoting melanoma cell growth and glucose metabolism, possibly through activation of the $M Y C$ target pathway. Additionally, DTL may serve as a potential therapeutic target for the future development of drugs for melanoma treatment.

\section{Acknowledgments}

Funding: This work was sponsored by grants from the National Natural Science Foundation of China (81872522, 82073429), Youth Program of National Natural Science Foundation of China (82003335), Innovation Program of Shanghai Municipal Education Commission (2019-01-0700-07-E00046), the Program of Science and Technology Commission Shanghai Municipality (18140901800), Excellent Subject Leader Program of Shanghai Municipal Commission of Health and Family Planning (2018BR30), Clinical Research Program of Shanghai Hospital Development Center (SHDC2020CR1014B, SHDC12018X06), and Program of Shanghai Academic Research Leader (20XD1403300).

\section{Footnote}

Reporting Checklist: The authors have completed the MDAR reporting checklist. Available at https://atm.amegroups. com/article/view/10.21037/atm-21-6648/rc

Data Sharing Statement: Available at https://atm.amegroups. com/article/view/10.21037/atm-21-6648/dss 
Conflicts of Interest: All authors have completed the ICMJE uniform disclosure form (available at https://atm. amegroups.com/article/view/10.21037/atm-21-6648/coif). The authors have no conflicts of interest to declare.

Ethical Statement: The authors are accountable for all aspects of the work in ensuring that questions related to the accuracy or integrity of any part of the work are appropriately investigated and resolved. The study was conducted in accordance with the Declaration of Helsinki (as revised in 2013).

Open Access Statement: This is an Open Access article distributed in accordance with the Creative Commons Attribution-NonCommercial-NoDerivs 4.0 International License (CC BY-NC-ND 4.0), which permits the noncommercial replication and distribution of the article with the strict proviso that no changes or edits are made and the original work is properly cited (including links to both the formal publication through the relevant DOI and the license). See: https://creativecommons.org/licenses/by-nc-nd/4.0/.

\section{References}

1. Rebecca VW, Somasundaram R, Herlyn M. Pre-clinical modeling of cutaneous melanoma. Nat Commun 2020;11:2858.

2. Higa LA, Banks D, Wu M, et al. L2DTL/CDT2 interacts with the CUL4/DDB1 complex and PCNA and regulates CDT1 proteolysis in response to DNA damage. Cell Cycle 2006;5:1675-80.

3. Sansam CL, Shepard JL, Lai K, et al. DTL/CDT2 is essential for both CDT1 regulation and the early G2/M checkpoint. Genes Dev 2006;20:3117-29.

4. Ueki T, Nishidate T, Park JH, et al. Involvement of elevated expression of multiple cell-cycle regulator, DTL/RAMP (denticleless/RA-regulated nuclear matrix associated protein), in the growth of breast cancer cells. Oncogene 2008;27:5672-83.

5. Chen YC, Chen IS, Huang GJ, et al. Targeting DTL induces cell cycle arrest and senescence and suppresses cell growth and colony formation through TPX2 inhibition in human hepatocellular carcinoma cells. Onco Targets Ther 2018;11:1601-16.

6. Baraniskin A, Birkenkamp-Demtroder K, Maghnouj A, et al. MiR-30a-5p suppresses tumor growth in colon carcinoma by targeting DTL. Carcinogenesis 2012;33:732-9.
7. Perez-Peña J, Corrales-Sánchez V, Amir E, et al. Ubiquitin-conjugating enzyme E2T (UBE2T) and denticleless protein homolog (DTL) are linked to poor outcome in breast and lung cancers. Sci Rep 2017;7:17530.

8. Pan HW, Chou HY, Liu SH, et al. Role of L2DTL, cell cycle-regulated nuclear and centrosome protein, in aggressive hepatocellular carcinoma. Cell Cycle 2006;5:2676-87.

9. Yang L, Dai J, Ma M, et al. Identification of a functional polymorphism within the 3 -untranslated region of denticleless E3 ubiquitin protein ligase homolog associated with survival in acral melanoma. Eur J Cancer 2019;118:70-81.

10. Benamar M, Guessous F, Du K, et al. Inactivation of the CRL4-CDT2-SET8/p21 ubiquitylation and degradation axis underlies the therapeutic efficacy of pevonedistat in melanoma. EBioMedicine 2016;10:85-100.

11. Leone RD, Powell JD. Metabolism of immune cells in cancer. Nat Rev Cancer 2020;20:516-31.

12. Sullivan MR, Mattaini KR, Dennstedt EA, et al. Increased Serine Synthesis Provides an Advantage for Tumors Arising in Tissues Where Serine Levels Are Limiting. Cell Metab 2019;29:1410-21.e4.

13. Tasdogan A, Faubert B, Ramesh V, et al. Metabolic heterogeneity confers differences in melanoma metastatic potential. Nature 2020;577:115-20.

14. Cascone T, McKenzie JA, Mbofung RM, et al. Increased Tumor Glycolysis Characterizes Immune Resistance to Adoptive T Cell Therapy. Cell Metab 2018;27:977-87.e4.

15. Ratnikov BI, Scott DA, Osterman AL, et al. Metabolic rewiring in melanoma. Oncogene 2017;36:147-57.

16. Houles T, Gravel SP, Lavoie G, et al. RSK Regulates PFK2 Activity to Promote Metabolic Rewiring in Melanoma. Cancer Res 2018;78:2191-204.

17. Dai W, Xu X, Li S, et al. SOX4 Promotes Proliferative Signals by Regulating Glycolysis through AKT Activation in Melanoma Cells. J Invest Dermatol 2017;137:2407-16.

18. Bristot IJ, Kehl Dias C, Chapola H, et al. Metabolic rewiring in melanoma drug-resistant cells. Crit Rev Oncol Hematol 2020;153:102995.

(English Language Editor: J. Jones)

Cite this article as: $\mathrm{Lu} \mathrm{JJ}$, Chen FJ, Li Y, Xu X, Peng C, Yu N, Su LN, Tang L. DTL promotes melanoma progression through rewiring cell glucose metabolism. Ann Transl Med 2022;10(2):68. doi: 10.21037/atm-21-6648 